\title{
Direct Multiple Shooting Transcription with Polynomial Algebra for Optimal Control Problems Under Uncertainty
}

\author{
Cristian Greco $^{\mathrm{a}, *}$, Marilena Di Carlo ${ }^{\mathrm{a}}$, Massimiliano Vasile ${ }^{\mathrm{a}}$, Richard Epenoy ${ }^{\mathrm{b}}$ \\ ${ }^{a}$ Aerospace Centre of Excellence, University of Strathclyde, 75 Montrose Street, G1 1 XJ, \\ Glasgow, United Kingdom. \\ ${ }^{b}$ Centre National d'Etudes Spatiales (CNES), 18 Avenue Edouard Belin, 31401 Toulouse, \\ France.
}

\begin{abstract}
This paper proposes a novel approach to the solution of optimal control problems under uncertainty (OCPUUs). OCPUUs are first cast in a general formulation that allows the treatment of uncertainties of different nature, and then solved with a new direct transcription method that combines multiple shooting with generalised polynomial algebra to model and propagate extended sets. The continuity conditions on extended sets at the boundary of two adjacent segments are directly satisfied by a bounding approach. The Intrusive Polynomial Algebra aNd Multiple shooting Approach (IPANeMA) developed in this work can handle optimal control problems under a wide range of uncertainty models, including nonparametric, epistemic, and imprecise probability ones. In this paper, the approach is applied to the design of a robust low-thrust trajectory to a Near-Earth Object with uncertain initial conditions. It is shown that the new method provides more robust and reliable trajectories than the solution of an analogous deterministic optimal control problem.

Keywords: Optimal control under uncertainty, Robust control, Generalised multiple shooting, Intrusive polynomial algebra, Low-thrust trajectory optimisation
\end{abstract}

\footnotetext{
* Corresponding author

Email address: c.greco@strath.ac.uk (Cristian Greco)
} 


\section{Introduction}

Standard optimal control problems aim at finding the control law that optimally brings a given system to a desired state 1. However, the underlying assumption is that no uncertainty is affecting the system, its dynamics and 5 boundary conditions. Hence, the resulting control law, though optimal, might not be robust against the uncertainty naturally affecting the system. Uncertainty comes in different forms and can represent random phenomena, imperfect state knowledge, unknown model parameters or partially known dynamics. Furthermore, in nonlinear systems, even small deviations from the desired initial conditions can translate into significant differences in the terminal states.

In the specific case of low-thrust trajectories (i.e. trajectories whose dynamics is controlled via a thrust action that is small compared to the local gravity field) the limited control authority offered by the propulsion system makes correction to uncertainty in initial or terminal states challenging. If uncertainty

15 is not properly taken into account during the design of the trajectory and the associated control law, there can be no margin to compensate for possible deviations from the desired states. One common cause of trajectory deviation in low-thrust trajectories is missed-thrust due to sub-systems partial failure or external causes, like experienced by the Dawn 2 and Hayabusa missions 3 , 4 .

A common practical solution to compensate for possible deviations is to consider propellant margins and enforced coasting arcs in the reference trajectory design [5]. On the other hand, several authors have developed methods to directly incorporate uncertainty in the optimal control formulation. For example, methods based on model predictive control or closed-loop feedback control directly take into account correction terms based on possible deviations from the desired state 6. 7]. A method based on Taylor polynomials algebra was recently developed to deal with uncertain boundary conditions around a reference trajectory $[8$ and produce a robust guidance law. In addition, stochastic differential dynamic programming has been applied to space trajectory optimisation with uncertainty with an expected value formulation using the unscented 
transform [9].

The common assumption underneath these methods is the existence of a desired reference trajectory. Uncertainty is then producing an undesired deviation from it. This problem statement can be either formulated implicitly, by working with the mean value of the objective and constraints, or explicitly, by trying to compensate for the deviations. Furthermore, often these techniques can deal only with simple families of precise probability density distributions to represent uncertainty.

This paper proposes a method for the transcription and solution of stochastic optimal control problems with uncertainty phrased under a more general framework. First, optimal control problems under uncertainty are recast in a general framework that is suitable to model a wide class of stochastic problems including the case of non-parametric distributions and epistemic uncertainty.

Moreover, the premise of a reference trajectory is abandoned in favor of an extended uncertainty set representation. Each sample within the uncertainty set is a fully admissible pointwise trajectory with associated probability density. The objective function and constraints are framed under an expectation operator, which will be shown to be tailorable to different forms, including, but not limited to, objective and constraints in expected value, in probability and in standard deviation.

A novel generalised multiple shooting method is developed for the transcription of the infinite-dimensional optimal control problem under uncertainty into a finite-dimensional nonlinear constrained optimisation one. This novel transcription scheme employs generalised intrusive polynomial algebra [10 for the uncertainty propagation. This expansion in polynomial series generalises the more common use of Taylor polynomials (generally found in Differential Algebra or Jet Transports) and allows the use of any polynomial representations. With this extension, one can exploit the properties offered by polynomials other than Taylor, like min-max or uniform convergence. In this paper, states are ex60 panded in Chebyshev polynomials that were shown to exhibit a superior global convergence and robustness in a number of aerospace applications [11, 12]. 
Finally, the formulation and the novel approach are applied to the optimisation under uncertainty of an interplanetary low-thrust transfer to the asteroid Apophis. Uncertainty is introduced in the hyperbolic excess velocity at departure from Earth, and both the cases of precise probability models and epistemic uncertainty are considered. It will be shown that the solution produced with the approach proposed in this paper is more robust and reliable against the possible realisations of the uncertain parameters.

The structure of the remainder of the paper is as follows. Section 2 introduces the formulation of the addressed optimal control problem under uncertainty. Section 3 presents the main development, first introducing the intrusive polynomial algebra propagation, and then integrating it with the novel generalised multiple shooting framework using an expectation formulation for the objective function and constraints. Within this framework, a specific approach

75 is proposed based on polynomial reinitialisation and successive sampling. The developed tool is then applied to the optimisation of a low-thrust rendezvous trajectory to a Near-Earth Object in Section 4 . Finally, Section 5 concludes the paper with the final remarks.

\section{Optimal Control Under Uncertainty}

In the general Bolza formulation, a deterministic optimal control problem reads as

$$
\begin{array}{ll}
\min _{\mathbf{u}(t) \in U} & J=\Phi\left(t_{f}, \mathbf{x}_{f}\right)+\int_{t_{0}}^{t_{f}} L(t, \mathbf{x}, \mathbf{u}) d t \\
\text { s.t. } \quad & \dot{\mathbf{x}}=f(t, \mathbf{x}, \mathbf{u}, \mathbf{d}) \\
& \mathbf{g}(t, \mathbf{x}, \mathbf{u}, \mathbf{d}) \in \boldsymbol{G} \\
& \boldsymbol{\psi}\left(t_{0}, \mathbf{x}_{0}, t_{f}, \mathbf{x}_{f}\right) \in \mathbf{\Psi}
\end{array}
$$

so where $t$ denotes the independent variable, i.e. time in this paper, $\mathbf{x}$ the state variable, $\mathbf{u}$ the control and $\mathbf{d}$ the static parameters. The aim of the deterministic optimal control problem is to find an optimal control law $\mathbf{u}^{*}(t) \in U$ such that objective function $J$ in Equation [1a) is minimised while fulfilling, respectively, 
the dynamical nonlinear equations of motion in Equation (1b), the path constraints in Equation (1c), and the boundary conditions in Equation (1d). Path and boundary constraints are expressed with a set inclusion condition that represents both equality and inequality constraints. This formulation is suitable for the optimisation of a deterministic trajectory governed by a single control profile and a deterministic dynamics.

When uncertainties come into play, a set of admissible trajectories is associated to a single control profile. Hence, problem (1) needs to be extended to allow the treatment of a general optimal control problem for a dynamical system under uncertainty.

First we will consider uncertainty to be purely aleatory. In this case the uncertain parameter vector $\boldsymbol{\xi}$ is associated to a random variable $\mathbf{Z}$ with probability density function $p(\boldsymbol{\xi})$ and sample space $\Omega_{\boldsymbol{\xi}}$. The vector $\boldsymbol{\xi}$ includes both initial states and model parameters. The random variable $\mathbf{Z}$ induces the initial state $\mathbf{X}_{0}$ and model parameters $\mathbf{D}$ to be random variables as well, with density functions $p\left(\mathbf{x}_{0}\right)$ and $p(\mathbf{d})$, and sample spaces $\Omega_{\mathbf{x}_{0}}$ and $\Omega_{\mathbf{d}}$. On the other hand, the control variables will be treated as a completely deterministic input, since possible disturbances on the control can be modelled in the dynamics as a multiplicative noise incorporated in $\mathbf{Z}$. The dynamical nature of the system induces the state at time $t$ to be a random variable $\mathbf{X}(t)$ as well.

In the general nonlinear case, directly computing the evolution of the density distribution over time $p(\mathbf{x}(t))$ is an ambitious and, when possible, laborious task. Therefore, this problem is often tackled with sampling techniques. Indeed, the dynamical equations can be directly used as a map from the state and parameter sample space at a given time to the state sample space at another time. The distribution at the time of interest may be then reconstructed according to the sample responses, usually by fitting a parametric distribution.

In the context of optimal control under uncertainty, the definition of objective and constraint functions is critical because their formulation greatly affects the result and its interpretation. Common choices in stochastic programming are to formulate objective and constraints either in expected value or in proba- 
bility [13]. In this paper we propose the following unified formulation in expectation, that makes use of the auxiliary function $\phi$ of the generic random variable Z:

$$
\mathbb{E}[\phi(\mathbf{Z})]=\int_{\Omega_{\boldsymbol{\xi}}} \phi(\boldsymbol{\xi}) p(\boldsymbol{\xi}) d \boldsymbol{\xi} .
$$

The expectation formulation in (2) allows one to define a variety of different quantities by selecting the appropriate function $\phi$. Depending on the quantity of interest, the function $\phi$ can represent an objective or a constraint.

In the following we show how the expectation formulation encloses common alternatives for both the objective or constraints functions under uncertainty:

- Expected Value formulation: by definition, the expectation operator of a generic function of a random variable returns the expected value of that function. For example, for a constraint on the expected value of the uncertain terminal state, the auxiliary function $\phi$ is the identity mapping of the final state:

$$
\phi_{\psi}\left(t_{f}, \mathbf{X}_{f}\right)=\mathbf{X}_{f},
$$

and the constraint is:

$$
\mathbb{E}\left[\mathbf{X}_{f}\right]=\overline{\mathbf{x}}_{f}
$$

- Probability formulation: the probability of an event is the expectation of the indicator function of such event. For example, for a chance constraint on the uncertain terminal state to reach the target region $A$ with probability larger than or equal to $1-\rho$, the auxiliary function $\phi$ is defined as

$$
\phi_{\psi}\left(t_{f}, \mathbf{X}_{f}\right)=\mathbb{I}_{A}\left(\mathbf{X}_{f}\right),
$$

where

$$
\mathbb{I}_{A}\left(\mathbf{X}_{f}=\mathbf{x}_{f}\right)= \begin{cases}1 & \text { if } \mathbf{x}_{f} \in A \\ 0 & \text { if } \mathbf{x}_{f} \notin A\end{cases}
$$

From here, the constraint is formulated as

$$
\mathbb{E}\left[\mathbb{I}_{A}\left(\mathbf{X}_{f}\right)\right]=\operatorname{Pr}\left(\mathbf{X}_{f} \in A\right) \in \mathbf{\Phi}_{\psi}=[1-\rho, 1] ;
$$


- Higher-order moments formulation: by definition, the $n$-th moment of a probability density function is the expectation of its n-th power. For example, for a constraint on the variance of the final state, the auxiliary function $\phi$ is defined as

$$
\phi_{\boldsymbol{\psi}}\left(t_{f}, \mathbf{X}_{f}\right)=\left(\mathbf{X}_{f}-\mathbb{E}\left[\mathbf{X}_{f}\right]\right)\left(\mathbf{X}_{f}-\mathbb{E}\left[\mathbf{X}_{f}\right]\right)^{T}
$$

From here, the covariance is obtained as

$$
\mathbb{E}\left[\left(\mathbf{X}_{f}-\mathbb{E}\left[\mathbf{X}_{f}\right]\right)\left(\mathbf{X}_{f}-\mathbb{E}\left[\mathbf{X}_{f}\right]\right)^{T}\right]=\operatorname{cov}\left[\mathbf{X}_{f}, \mathbf{X}_{f}\right]
$$

and the relevant constraint can be imposed.

The same flexibility holds for different definitions of the objective function under uncertainty.

We can now use the formulation in expectation to cast a general optimal control problem under uncertainty in the following form:

$$
\begin{array}{ll}
\min _{\mathbf{u}(t) \in U} & \mathbb{E}\left[\phi_{J}(t, \mathbf{X}, \mathbf{u}, \mathbf{D})\right] \\
\text { s.t. } & \dot{\mathbf{X}}=f(t, \mathbf{X}, \mathbf{u}, \mathbf{D}) \\
& \mathbb{E}\left[\phi_{\mathbf{g}}(t, \mathbf{X}, \mathbf{u}, \mathbf{D})\right] \in \mathbf{\Phi}_{\mathbf{g}} \\
& \mathbb{E}\left[\phi_{\boldsymbol{\psi}}\left(t_{0}, \mathbf{X}_{0}, t_{f}, \mathbf{X}_{f}\right)\right] \in \mathbf{\Phi}_{\boldsymbol{\psi}} .
\end{array}
$$

Generally problem 10 does not have a closed form solution. Thus a numerical solution scheme is required. The next section will introduce a novel numerical method for the solution optimal control problems under uncertainty in the form of problem 10 .

\section{Direct Multiple Shooting with Generalised Polynomial Algebra}

A well established method for the solution of optimal control problems is known as direct multiple shooting [14. Direct multiple shooting starts by discretising the time domain into time segments $\left[t_{i}, t_{i+1}\right]$. Each segment is defined by an initial state $\mathbf{x}_{i}$ and a parametric function $\mathcal{U}_{i}\left(t, \boldsymbol{\beta}_{i}\right)$ of some free parameters 
$\boldsymbol{\beta}_{i}$, such that the control profile on each segment is defined as $\mathbf{u}_{i}(t)=\mathcal{U}_{i}\left(t, \boldsymbol{\beta}_{i}\right)$. A Non-Linear Programming (NLP) solver is then used to identify the optimal value of the states $\mathbf{x}_{i}$ and parameters $\boldsymbol{\beta}_{i}$. Every time the NLP solver assigns a value to $\mathbf{x}_{i}$ and $\boldsymbol{\beta}_{i}, \mathbf{x}_{i}$ is propagated from $t_{i}$ to $t_{i+1}$. Continuity constraints on the states at the boundary of two adjacent segments are enforced by the NLP solver to ensure the continuity of the final solution and the satisfaction of the boundary conditions. Once all the segments are propagated the values of the objective function and constraints are computed.

When uncertainties are introduced, the pointwise state $\mathbf{x}_{i}$ becomes a set and thus a propagation method that can handle sets of values is required. In Section 3.1. we propose the use of Generalised Polynomial Algebra (GPA) to model and propagate the state variables as functions of the uncertain variables [15, 10]. Section 3.2 introduces GPA in a direct multiple shooting scheme to solve problem (10). The resulting approach is named IPANeMA (Intrusive Polynomial Algebra aNd Multiple shooting Approach).

\subsection{Uncertainty Propagation}

Consider the set $\Omega_{\mathbf{x}_{0}}$ of all the possible values of the state variable $\mathbf{x}$ at time $t_{0}$, induced by all the possible values of the uncertain vector $\boldsymbol{\xi}$ over the compact set $\Omega_{\boldsymbol{\xi}}$, and a polynomial of degree $q$ in $n_{\xi}$ variables $P_{\mathbf{x}_{0}(\boldsymbol{\xi})} \in T_{q, n_{\xi}}$, where $T_{q, n_{\xi}}$ is a polynomial space. We are now interested in the evolution in time of the set $\Omega_{\mathbf{x}_{0}}$ so that we can calculate the propagated set $F_{t}(\boldsymbol{\xi})$ at any point in time. Note that if $\mathbf{x}$ was not a continuous function of $\boldsymbol{\xi}$ we could still consider a set $\hat{\Omega}_{\mathbf{x}_{0}}$ of a continuous function $\hat{\mathbf{x}}$ of $\boldsymbol{\xi}$ that contains all the realisation of $\mathbf{x}$ induced by $\boldsymbol{\xi}$.

In other words we propagate a set, with our polynomial model, that we assume to be compact and finite (we made this explicit). Once the set is propagated we compute the relevant statistical quantities by sampling the polynomials according to the distribution of $\xi$. As long as the dynamics is integrable for all values in the set we propagate no additional assumption on $\xi$ is required when we propagate the set. 
We formally define the set $F_{t}(\boldsymbol{\xi})$ as follows. For a given realisation of the uncertain vector $\boldsymbol{\xi}$ and a control law $\overline{\mathbf{u}}$ the propagated state $\mathbf{x}(t)$ at time $t$ is:

$$
\mathbf{x}(t, \boldsymbol{\xi})=\mathbf{x}_{0}(\boldsymbol{\xi})+\int_{t_{0}}^{t} f\left(\tau, \mathbf{x}_{0}(\boldsymbol{\xi}), \overline{\mathbf{u}}, \mathbf{d}(\boldsymbol{\xi})\right) d \tau,
$$

and the set $F_{t}(\boldsymbol{\xi})$ is:

$$
F_{t}(\boldsymbol{\xi})=\left\{\mathbf{x}(t, \boldsymbol{\xi}) \mid \forall \boldsymbol{\xi} \in \Omega_{\boldsymbol{\xi}}\right\}
$$

Here the assumption is that the dynamics $f$ is integrable for all values of $\boldsymbol{\xi} \in \Omega_{\boldsymbol{\xi}}$ and $\tau \in\left[t_{0}, t\right]$.

We now approximate the set $\Omega_{\mathbf{x}_{0}(\boldsymbol{\xi})}$ with the polynomial $P_{\mathbf{x}}\left(t_{0}\right)=P_{\mathbf{x}_{0}(\boldsymbol{\xi})}$ and propagate this approximation forward in time to obtain:

$$
F_{t}(\boldsymbol{\xi}) \approx \widetilde{F}_{t}(\boldsymbol{\xi})=P_{\mathbf{x}}(t, \boldsymbol{\xi})=\sum_{j=1}^{\mathcal{N}} \alpha_{j}(t) P_{j}(\boldsymbol{\xi}),
$$
ated coefficient. We propose to compute the polynomial approximation $\widetilde{F}_{t}(\boldsymbol{\xi})$ with Generalised Polynomial Algebra first introduced in [1] and then further developed in [12] and 10. Note that $P_{\mathbf{x}_{0}(\boldsymbol{\xi})}$ does not represent a stochastic process or a random variable but a set of states that are a function of $\boldsymbol{\xi}$.

GPA starts by expressing the generic polynomial $P_{\mathbf{x}_{0}(\boldsymbol{\xi})}$ into monomial bases so that $P_{\mathbf{x}_{0}(\boldsymbol{\xi})} \in T_{q, n_{\xi}}^{\prime}$, where $T_{q, n_{\xi}}^{\prime}$ is the functional space of the polynomial in monomial bases. A set of algebraic operations between polynomials in $T_{q, n_{\xi}}^{\prime}$ is then introduced defining an algebra in $T_{q, n_{\xi}}^{\prime}$. Denoting with $V$ and $W$ the multivariate polynomial expansions of two functions $v$ and $w$, the algebraic operation $\oplus=\{+,-, \cdot, /\}$ between real-valued functions has its correspondent $\otimes$ in the polynomial space:

$$
v(\boldsymbol{\xi}) \oplus w(\boldsymbol{\xi}) \sim V(\boldsymbol{\xi}) \otimes W(\boldsymbol{\xi}) \in T_{q, n_{\xi}}^{\prime} .
$$

165

The result of the addition (or equivalently subtraction) of two elements of $T_{q, n_{\xi}}^{\prime}$ is still an element of the same functional space. In order to keep the order of the polynomials constant, the result of multiplication is truncated to the order 
$q$ and so is the result of any composition. Composition rules are defined to handle division and other elementary functions such as trigonometric functions, exponents, logarithms, etc. These polynomial operations are implemented using the $\mathrm{C}++$ overloading operator within the Strathclyde Mechanical and Aerospace Research Toolbox for Uncertainty Quantification [12].

Given this set of operations, any integrator for the propagation of ordinary differential equations can be directly applied to the propagation of $\Omega_{\mathbf{x}_{0}(\boldsymbol{\xi})}$ through the dynamical system constraints in Equation $10 \mathrm{~b}$ once $\Omega_{\mathbf{x}_{0}(\boldsymbol{\xi})}$ is expressed as an element of $T_{q, n_{\xi}}^{\prime}$ (for more details and examples refer to [10]).

During propagation, the uncertain model parameters are handled similarly to the initial conditions. The parameter sample domain $\Omega_{\mathbf{d}}$, induced by the random variable $\mathbf{Z}$, is bounded by a constant multivariate polynomial $P_{\mathbf{d}} \in T_{q, n_{\xi}}$, which is composed in $P_{\mathbf{x}}(t)$ through the dynamical operations.

Once $\widetilde{F}_{t}(\boldsymbol{\xi})$ is available, one can compute the expectation of a generic function of the propagated uncertain state as

$$
\mathbb{E}[\phi(t, \mathbf{X})]=\mathbb{E}\left[\phi\left(t, \widetilde{F}_{t}(\mathbf{Z})\right)\right]=\int_{\Omega_{\boldsymbol{\xi}}} \phi\left(t, \widetilde{F}_{t}(\boldsymbol{\xi})\right) p(\boldsymbol{\xi}) d \boldsymbol{\xi} .
$$

\subsection{IPANeMA}

The goal of the direct transcription method proposed in this paper is to evaluate the expectations of objective function and constraints in problem 10 resulting from a given control $\overline{\mathbf{u}}(t)$.

The standard direct multiple shooting scheme is modified so that GPA is used to propagate the control law and the effect of uncertainty on each time segment $\left[t_{i}, t_{i+1}\right]$. However, the direct application of GPA to propagate states and controls poses two key problems:

- how to match the states at the boundaries of each time segment

- how to define the dependence of the states on the uncertain parameters on each time segment

The naive approach to solve the latter problem would be to express the dependence of the states of each segment on the uncertainty set $\Omega_{\xi}$. In fact one 
could solve both the problems by using GPA to perform a single integration from initial to final time to get:

$$
\widetilde{F}_{t}(\boldsymbol{\xi})=P_{\mathbf{x}}(t, \boldsymbol{\xi})
$$

The advantage of this approach is that the numerical uncertainty propagation has to be performed only once, and then the expectation can be computed by means of an inexpensive polynomial evaluation.

Since the propagation is performed directly from the initial to the final time, this scheme can be seen as a generalised single shooting transcription. However, this approach implies a polynomial representation of $n_{\xi}=n_{s}+n_{d}$ uncertain variables, where $n_{s}$ is the size of the state vector and $n_{d}$ is the total number of uncertain model parameters. Since the number of terms in the polynomial expansion grows with $\left(n_{\xi}+q\right) ! /\left(n_{\xi} ! q !\right)$, this approach can only handle a limited number of uncertain model parameters. When different (and independent) uncertain parameters affect the system evolution at different instants of time (e.g. multi-phase trajectories, discretised control with disturbances, etc.), the size of the polynomial would quickly become intractable.

Consider now the uncertain model parameter vector to be defined as follows:

$$
\mathbf{d}=\left[\mathbf{d}_{0}, \mathbf{d}_{1}, \ldots, \mathbf{d}_{i}, \ldots\right]
$$

such that the realisations of the uncertain model parameters $\mathbf{d}_{i}$ manifest only over the time interval $\left[t_{i}, t_{i+1}\right]$. From here, the goal is to develop a transcription method such that GPA can be applied to each sub-segment independently of the uncertain model parameters affecting the other segments. Consequently the algebra dimension in the $i$-th segment reduces to $n_{\xi_{i}}=n_{s}+n_{d_{i}}$, namely the number of the uncertain state variables $\mathbf{X}\left(t_{i}\right)$ at the beginning of the segment and the number of uncertain model parameters $\mathbf{d}_{i}$ producing realisations over $t \in\left[t_{i}, t_{i+1}\right]$. With this scheme, the polynomial $P_{\mathbf{x}_{i}}$ representing the uncertain states at $t_{i}$ is propagated to $P_{\mathbf{x}_{i+1}}$ at $t_{i+1}$ only under the effect of uncertain parameters $\mathbf{D}_{i}$. 
The main difficulty of the proposed discretisation arises from the necessity to impose continuity conditions between two hyper-dimensional uncertainty sets at the boundary of two adjacent segments.

If one calls $F_{t+1}^{(p)}$ the set of states at time $t+1$ and $F_{t+1}^{(g)}$ the superset $F_{t+1}^{(g)} \supseteq$ ${ }_{220} F_{t+1}^{(p)}$, the corresponding polynomial approximations become $P_{\mathbf{x}_{i+1}}^{(p)}$ and $P_{\mathbf{x}_{i+1}}^{(g)}$, where $P_{\mathbf{x}_{i+1}}^{(p)}$ is a function of $\mathbf{X}_{i}$ and $\mathbf{D}_{i}$, and $P_{\mathbf{x}_{i+1}}^{(g)}$ is what we call the reinitialised set of uncertain states $\mathbf{X}_{i+1}$ at time $t+1$. Note that the stochastic vector $\mathbf{D}_{i+1}$ has realisation only over the open interval $\left(t_{i+1}, t_{i+2}\right]$.

Hence, the propagation phase is carried out as described in Algorithm 1 and is represented in the cartoon in Figure 1 for a two-dimensional example. In Figure 1 the initial uncertainty set expanded in polynomial basis $P_{\mathbf{x}_{0}}$ at $t_{0}$ is propagated through the dynamical system with the polynomial algebra to $P_{\mathbf{x}_{1}}^{(p)}$ at the first segment final time $t_{1}$. In order to obtain an uncertainty representation function of the variables of the next segment only, the uncertain region $P_{\mathbf{x}_{1}}^{(p)}$ is reinitialised as a hyper-cube $P_{\mathbf{x}_{1}}^{(g)}$ which conservatively bounds the propagated set. The propagation and reinitialisation steps are repeated for each multiple shooting segment.

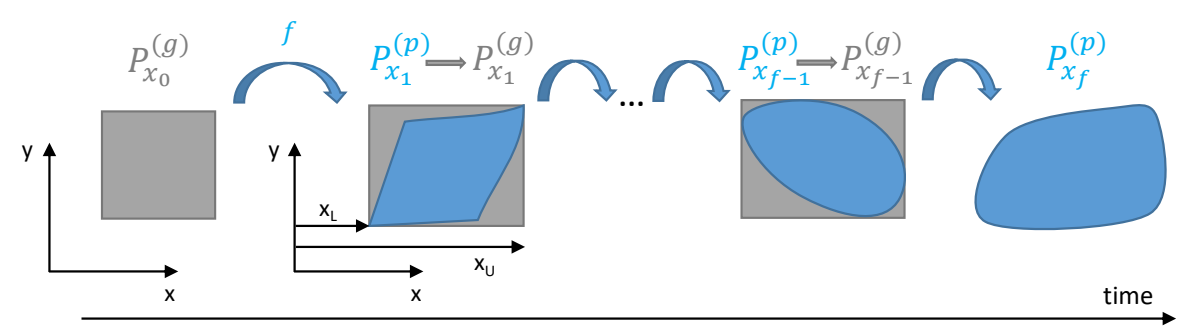

Figure 1: Graphical sketch of intrusive polynomial propagation approach for the generalised multiple shooting. The grey boxes represent the reinitalisation hyper-boxes, whereas the blue regions depict the propagated polynomials.

By outer bounding the propagated region, the key advantage is that all the possible state realisations are included, thus granting the continuity of any pointwise trajectory realisation. This simple approach comes at the expense of 


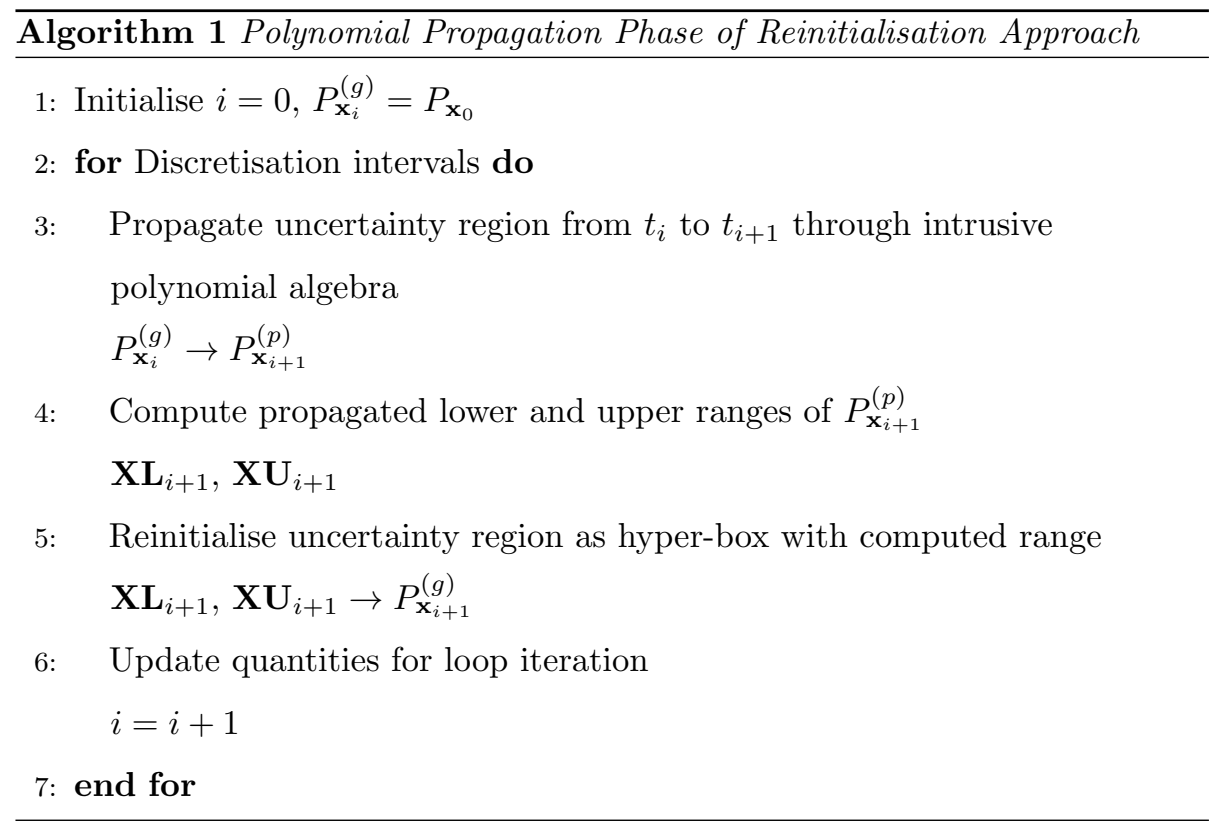

propagating larger regions than strictly needed.

It is worth noting that the state uncertainty region can be propagated at any intermediate time of interest $t \in\left[t_{i}, t_{i+1}\right]$ without the need of further discretisation and reinitialisation. Trivially, an intermediate polynomial $P_{\mathbf{x}_{t}}^{(p)}$ can be saved during the propagation phase. Therefore, the result of this propagation approach is a chain of polynomial surrogates describing the uncertain state $\mathbf{X}_{t}$ as a recursive function of the initial conditions $\mathbf{X}_{0}$ and the uncertain parameters $\mathbf{D}_{0: i}=\left[\mathbf{D}_{0}, \ldots, \mathbf{D}_{i}\right]$ which affected the system evolution up to time $t \leq t_{i+1}$. At this step however, the hyper-box reinitialisation caused the 245 uncertain state polynomial representation to be an over-estimation of the true uncertainty space.

An estimation of the correct size of the actual propagated set $F_{t}$ is recovered by sampling $P_{\mathbf{x}_{t}}^{(p)}$. The approach to propagate samples and recover the actual state uncertainty region is described in Algorithm 2, and graphically represen250 tated in Figure 2. The approach starts by drawing $N$ state samples $\mathbf{x}_{0}^{(j)}$ from the initial uncertainty state space and $N$ parameter samples $\mathbf{d}_{0}^{(j)}$ from the first 
segment uncertainty parameter space. Each of the $N$ pairs $\left(\mathbf{x}_{0}^{(j)}, \mathbf{d}_{0}^{(j)}\right)$ corresponds to a deterministic realisation of the trajectory (black dots and lines in Figure 22, which is propagated using the polynomial mapping $P_{\mathbf{x}_{1}}^{(p)}$ computed 255 as in Algorithm 1 The propagated state $\mathbf{x}_{1}^{(j)}$ is scaled within the polynomial input domain, and new parameter samples $\mathbf{d}_{1}^{(j)}$ are drawn for the successive segment to form $N$ new pairs $\left(\mathbf{x}_{1}^{(j)}, \mathbf{d}_{1}^{(j)}\right)$. The propagation and sampling steps are iterated for each multiple shooting segment. Thanks to this approach, the true uncertainty region (green in Figure 2) is recovered from the conservative polynomial expansion (grey in Figure 2), and represented by a cloud of discrete samples.

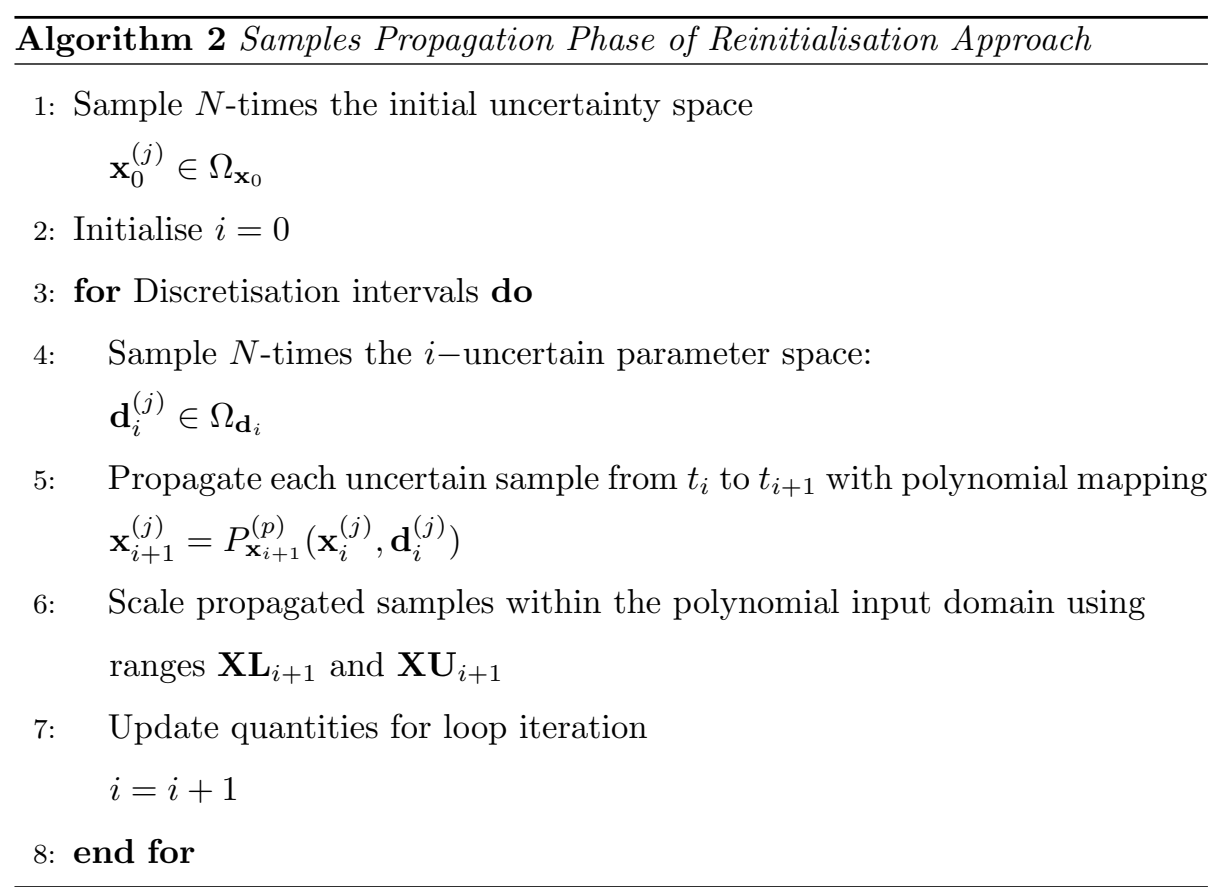

Hence, the general result of this approach is a pointwise recursive surrogate model summarised as

$$
\widetilde{F}_{t}(\boldsymbol{\xi})=P_{\mathbf{x}}(t, \boldsymbol{\xi})=P_{\mathbf{x}_{t}}^{(p)}\left(\ldots\left(P_{\mathbf{x}_{2}}^{(p)}\left(P_{\mathbf{x}_{1}}^{(p)}\left(\mathbf{x}_{0}, \mathbf{d}_{0}\right), \mathbf{d}_{1}\right), \ldots\right), \mathbf{d}_{i}\right),
$$

that maps the uncertain initial conditions and model parameters to the state vector at any time $t \in\left[t_{i}, t_{i+1}\right]$. 


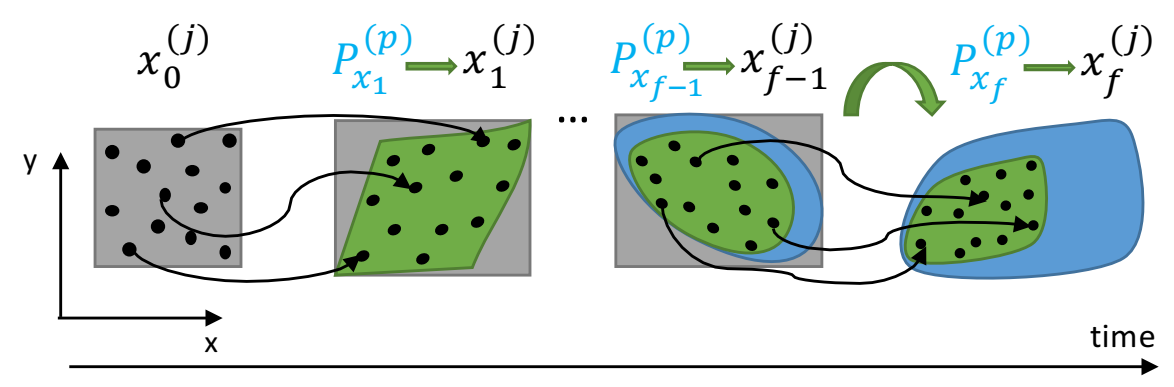

Figure 2: Graphical sketch of the recovery approach by sampling for the generalised multiple shooting. The grey boxes represent the reinitialisation hyper-boxes, the blue regions depict the propagated polynomials, while the green areas symbolise the true uncertainty regions reconstructed by the black samples.

The recursive polynomial formula (17) is the equation of the direct multiple shooting based on GPA. Formula 17 intrinsically guarantees continuity at the boundaries of each time interval and maintain the dimensionaly of the uncertainty space on each and any time interval equal to $n_{\xi_{i}}=n_{s}+n_{d_{i}}$. Note that the use of an outer set approximation removes the need of explicit defect constraints and free state variables at the start of each time interval, hence reducing the dimensionality of the associated constrained optimisation problem. The only free variables to be optimised are the control parameters in each sub-segment.

\subsubsection{Objective and Constraints Computation}

With the recursive polynomial mapping in Equation (17) the set of uncertain states can be recovered at any time $t \in\left[t_{0}, t_{f}\right]$ via the simple evaluation of a polynomial through sampling. These samples can be exploited to compute the general expectation form as in Equation (15). The expected value of objective and constraints can be computed with one of these two sample-based techniques:

- Direct Monte Carlo with samples $\boldsymbol{\xi}^{(j)}$ drawn directly from the uncertain probability distribution $p(\boldsymbol{\xi})$. In this case, the expected value approximated is

$$
\mathbb{E}\left[\phi\left(\widetilde{F}_{t}(\mathbf{Z})\right)\right] \approx \frac{1}{N} \sum_{j=1}^{N} \phi\left(\widetilde{F}_{t}\left(\boldsymbol{\xi}^{(j)}\right)\right)
$$


- Importance Sampling with samples $\boldsymbol{\xi}^{(j)}$ drawn from an auxiliary proposal distribution $\pi(\boldsymbol{\xi})$. In this case, the resulting approximation is

$$
\begin{aligned}
\int_{\Omega_{\boldsymbol{\xi}}} \phi\left(\widetilde{F}_{t}(\boldsymbol{\xi})\right) p(\boldsymbol{\xi}) d \boldsymbol{\xi} & =\int_{\Omega_{\boldsymbol{\xi}}} \frac{\phi\left(\widetilde{F}_{t}(\boldsymbol{\xi})\right) p(\boldsymbol{\xi})}{\pi(\boldsymbol{\xi})} \pi(\boldsymbol{\xi}) d \boldsymbol{\xi} \\
& \approx \frac{1}{N} \sum_{j=1}^{N} \frac{\phi\left(\widetilde{F}_{t}\left(\boldsymbol{\xi}^{(j)}\right)\right) p\left(\boldsymbol{\xi}^{(j)}\right)}{\pi\left(\boldsymbol{\xi}^{(j)}\right)}
\end{aligned}
$$

where $\pi(\boldsymbol{\xi})$ should be chosen such that its support is greater than or equal to the support of $p(\boldsymbol{\xi})$.

Hence, the sampling in steps 1 and 4 of Algorithm 2 can be performed either directly from the corresponding density function, or using an importance distribution. Then, the expectations, associated to the objective and constraints, should be computed plugging the propagated samples in the relevant formula.

The second proposed scheme should be preferred when the probability distribution is complex to sample but rather easy to evaluate, or when the expectation has to be evaluated for a set of possible density functions $p \in \mathcal{P}$, as in the case of epistemic uncertainty or an imprecise probability model [16]. Indeed, in the latter case, the most expensive evaluation $\phi\left(\widetilde{F}_{t}\left(\boldsymbol{\xi}^{(j)}\right)\right)$ should be performed only once per sample, and the expectation corresponding to different distributions is computed by using different weights $p\left(\boldsymbol{\xi}^{(j)}\right)$, which are generally inexpensive evaluation of the specific density function. This is a powerful generalisation for the developed scheme as it allows one to overcome the need of precise specification of a single uncertainty density function, which is a process often subject to simplifying and unsound assumptions, e.g. Gaussianity.

Clearly, other techniques could be applied to the computation of the expectation, but Monte Carlo methods have been selected because: they are not restricted to specific families of probability distributions; their convergence rate to the true expected value is independent of the uncertainty space dimensionality [17, making it appropriate for realistic practical applications.

Depending on the uncertain distribution and on the degree of nonlinearity of the equations of motion, the number of samples required to have an accurate 
estimation of the expectation may be rather high. Furthermore, the transcription is called numerous times during the optimisation process. Therefore, the employment of an inexpensive polynomial mapping in place of direct numerical propagation plays a crucial role in the practical application of IPANeMA to realistic scenarios.

From the approach presented it follows that the proposed shooting generalisation to the uncertain scenario is a natural extension of the classical deterministic transcription, where a single propagation is replaced by multiple trajectory realisations, which are then weighted according to the uncertainty probability distribution to compute the objective and constraints values.

\subsubsection{Representation of the Control Set}

In order to avoid a new polynomial propagation each time a new control law $\overline{\mathbf{u}}(t)$ is generated by the NLP solver, we expanded also the controls with GPA. Specifically, the set of control parameters $\Omega_{\boldsymbol{\beta}_{i}}$ can be approximated by a time-static multivariate polynomial $P_{\boldsymbol{\beta}_{i}} \in T_{q, n_{\xi_{i}}}^{\prime}$, where the number of uncertain variables $n_{\xi_{i}}=n_{s}+n_{d_{i}}+n_{\boldsymbol{\beta}_{i}}$ is increased accordingly. Then, the set of control profiles in each time interval descends from the parameter-control relationship $P_{\mathbf{u}_{i}}(t)=\mathcal{U}_{i}\left(t, P_{\boldsymbol{\beta}_{i}}\right)$, where the composition rules defined in the GPA apply. Once the expansion is computed, for each $\boldsymbol{\beta}_{i} \in \Omega_{\boldsymbol{\beta}_{i}}$, both the control profile $\mathbf{u}_{i}(t)=\mathcal{U}_{i}\left(t, \boldsymbol{\beta}_{i}\right)$ and the associated set of propagated states can be computed with a single polynomial evaluation. With this procedure, only one uncertainty polynomial propagation is needed, and it can be precomputed before the optimisation cycle. Consequently, the update of the set of propagated states for a new vector of the control parameters comes at a very low computational cost.

\subsubsection{Smoothing of the Indicator Function}

When a gradient-based method is employed to solve the NLP problem, one practical difficulty arises from the computation of objective and constraints in probability. Although probability constraints (and objectives) are an intuitive 
evaluation of the indicator function which is discontinuous in nature. This discontinuity introduces a significant difficulty when computing the derivatives. In the general case, in fact, one cannot guarantee the differentiability of the expectation with respect to the controls. Even more so when the expectation 335 is computed with a sample-based numerical technique (see Equations (18) or (19).

In order to overcome this difficulty, IPANeMA implements the option to substitute the indicator function with a smoother approximation obtained by convolution 13 .

Convolution is applied by first redefining the indicator function with the following membership condition: $\eta_{A}: \mathbb{R}^{n_{s}} \rightarrow \mathbb{R}$ such that:

$$
\left\{\begin{array}{l}
\left|\eta_{A}(\mathbf{X}=\mathbf{x})\right| \leq 1 \text { if } \mathbf{x} \in A \\
\left|\eta_{A}(\mathbf{X}=\mathbf{x})\right|>1 \text { if } \mathbf{x} \notin A
\end{array}\right.
$$

Hence, the indicator function can be equivalently expressed as

$$
\mathbb{I}_{A}(\mathbf{X})=\mathbb{I}_{[-1,+1]}\left(\eta_{A}(\mathbf{X})\right)
$$

Now, if we introduce the smoothing function $h$, for each state realisation $\mathbf{x}$, the convolution of the indicator function with $h$ results in:

$$
\begin{aligned}
\mathbb{I}_{[-1,+1]}^{(r)}\left(\eta_{A}(\mathbf{X}=\mathbf{x})\right) & =\int_{-\infty}^{+\infty} \mathbb{I}_{[-1,+1]}(y) \frac{1}{r} h\left(\frac{\eta_{A}(\mathbf{x})-y}{r}\right) d y \\
& =\int_{-1}^{+1} \mathbb{I}_{[-1,+1]}(y) \frac{1}{r} h\left(\frac{\eta_{A}(\mathbf{x})-y}{r}\right) d y
\end{aligned}
$$

with $r>0$ a small positive scaling parameter. The integration interval is restricted to the interval $[-1,+1]$ because of the membership function $\eta_{A}$. The smoothing function $h$ is chosen so that $h: \mathbb{R} \rightarrow \mathbb{R}, h$ is non-negative, symmetric, with a unique maximum in 0 , and integral equal to 1 over the interval $[-1,1]$. These properties imply $\lim _{r \rightarrow 0} h(\cdot / r) / r=\delta$, with $\delta$ the Dirac delta. Hence, for 345 $r \rightarrow 0$ the convolution result tends to the original indicator function [13].

Finally, the sampling grid used to compute the smoothed indicator function is kept constant within one major iteration of the NLP solver. This is particularly important when derivatives are approximated with finite differences in 
order to avoid introducing noise in the computation of the gradients of objective and constraints.

\subsubsection{Numerical Solution of the NLP Problem}

The NLP problem resulting from the transcription approach implemented in IPANeMA is dense and low-dimensional, as no intermediate state vectors and continuity constraints are present, as explained above, and the control paramimplementation, this NPL problem is solved using WORHP [18].

\section{Test case: Optimal-fuel Interplanetary Rendezvous}

In this section IPANeMA is applied to the robust optimisation of a lowthrust space trajectory. This test case aims at assessing the suitability and

performance of the approach implemented in IPANeMA when applied to the preliminary design of robust space trajectories.

\subsection{Mission Scenario}

The goal is to compute the propellant-optimal rendezvous to the near-Earth asteroid 99942 Apophis $\left(2004 \mathrm{MN}_{4}\right)$ with a low-thrust spacecraft departing from the Earth. The ephemerides of Earth and Apophis at different epochs are computed with NASA's SPICE through high-fidelity prediction routines [19]. The initial date of the interplanetary leg is $22 / 10 / 2026$ for a total time of flight of 628 days. The engine has a maximum thrust of $T_{\max }=53 \mathrm{mN}$, a specific impulse of $3080 \mathrm{~s}$, and an initial mass of $m_{0}=644.3 \mathrm{~kg}$.

The three-dimensional motion of the spacecraft is described with nonsingular 
equinoctial elements [20]:

$$
\begin{aligned}
& a \\
& P_{1}=e \sin (\Omega+\omega) \\
& P_{2}=e \cos (\Omega+\omega) \\
& Q_{1}=\tan (i / 2) \sin (\Omega) \\
& Q_{2}=\tan (i / 2) \cos (\Omega) \\
& L=\Omega+\omega+\theta
\end{aligned}
$$

where $[a, e, i, \Omega, \omega, \theta]$ are the standard Keplerian elements.

The governing equations of motion are the Gauss' planetary equations in a radial-transverse-normal (RTN) reference frame. The fast angular variable $L$, i.e. the true longitude, will be used as independent variable to replace the time. Under the assumption that the magnitude of the low-thrust control is significantly smaller than the local gravitational force, the resulting system of equations is 21]:

$$
\begin{aligned}
\frac{d a}{d L} & =\frac{2 a^{3} B^{2}}{\mu}\left[\frac{P_{2} \sin L-P_{1} \cos L}{\Phi^{2}(L)} f_{R}+\frac{1}{\Phi(L)} f_{T}\right] \\
\frac{d P_{1}}{d L} & =\frac{B^{4} a^{2}}{\mu}\left[-\frac{\cos L}{\Phi^{2}(L)} f_{R}+\left(\frac{P_{1}+\sin L}{\Phi^{3}(L)}+\frac{\sin L}{\Phi^{2}(L)}\right) f_{T}-P_{2} \frac{Q_{1} \cos L-Q_{2} \sin L}{\Phi^{3}(L)} f_{N}\right] \\
\frac{d P_{2}}{d L} & =\frac{B^{4} a^{2}}{\mu}\left[+\frac{\sin L}{\Phi^{2}(L)} f_{R}+\left(\frac{P_{2}+\cos L}{\Phi^{3}(L)}+\frac{\cos L}{\Phi^{2}(L)}\right) f_{T}++P_{1} \frac{Q_{1} \cos L-Q_{2} \sin L}{\Phi^{3}(L)} f_{N}\right] \\
\frac{d Q_{1}}{d L} & =\frac{B^{4} a^{2}}{2 \mu}\left(1+Q_{1}^{2}+Q_{2}^{2}\right) \frac{\sin L}{\Phi^{3}(L)} f_{N} \\
\frac{d Q_{2}}{d L} & =\frac{B^{4} a^{2}}{2 \mu}\left(1+Q_{1}^{2}+Q_{2}^{2}\right) \frac{\cos L}{\Phi^{3}(L)} f_{N}
\end{aligned}
$$

where $B=\sqrt{1-P_{1}^{2}-P_{2}^{2}}$ and $\Phi(L)=1+P_{1} \sin L+P_{2} \cos L$. The thrust vector is defined by its acceleration magnitude, azimuth angle and elevation angle in the RTN reference frame:

$$
\mathbf{f}=\left[\begin{array}{c}
f_{R} \\
f_{T} \\
f_{N}
\end{array}\right]=\left[\begin{array}{c}
\epsilon \sin \alpha \cos \beta \\
\epsilon \cos \alpha \cos \beta \\
\epsilon \sin \beta
\end{array}\right] .
$$


First we calculate a reference solution with no uncertainty which departures from Earth with the hyperbolic excess velocity reported in Table 1 where the vector is defined in terms of magnitude, azimuth and elevation in the Earthcentered inertial reference frame. In this deterministic case, the constraint is

Table 1: Deterministic hyperbolic excess velocity at departure from Earth.

\begin{tabular}{c|c}
\hline Component & Value \\
\hline$v_{\infty}^{\text {det }}$ & $3.34[\mathrm{~km} / \mathrm{s}]$ \\
$\alpha_{\infty}^{\text {det }}$ & $38.39[\mathrm{deg}]$ \\
$\beta_{\infty}^{\text {det }}$ & $-28.68[\mathrm{deg}]$ \\
\hline
\end{tabular}

imposed on the final state to exactly match the equinoctial elements of Apophis at the final time, and the objective function, to be minimised, is the low-thrust $\Delta V$. The reference trajectory was generated with FABLE (Fast Analytical Boundary-value Low-thrust Estimator) [22, which transcribes the optimal control problem into a sequence of coast and constant thrust arcs with variable length. The size of each thrust and cost arc is then optimised to minimise the objective function and satisfy the boundary conditions. In FABLE, the dynamics in Equation (24) is analytically propagated using a first-order expansion in the perturbing control acceleration [21]. The value of the objective function for the reference trajectory is $\Delta V=2.0318 \mathrm{~km} / \mathrm{s}$.

\subsection{Uncertainty Formulation}

In the following, we consider uncertainties stemming from the interplanetary orbit injection velocity vector. Uncertainty is modelled with the density functions defined in Table 2. Note that a support of $4-\sigma$ from the peak value is considered.

The uncertain vector, defined as $\boldsymbol{\xi}=\left[v_{\infty}^{u n c}, \alpha_{\infty}^{u n c}, \beta_{\infty}^{u n c},\right]^{T}$, induces uncertainty in the initial conditions. The resulting distributions of the equinoctial elements at initial time are displayed in Figure 3. The colour code indicates the probability density associated to each sample. In the same figures, the black dot is the expected value and the dashed line represents the projected $1 \sigma$ ellipsoid 
Table 2: Density functions modelling uncertainties on the hyperbolic excess velocity at departure from Earth. The deterministic excess velocity magnitude in Table 1 is the mode of the reversed Gaussian tail density distribution modelling the uncertainty on $v_{\infty}^{u n c}$, whereas the angular components are the mean of the Gaussian density distributions describing the uncertainty on $\alpha_{\infty}^{u n c}$ and $\beta_{\infty}^{u n c}$.

\begin{tabular}{c|l|c|c|c}
\hline Component & Density Function & Param. & Std. deviation & Support \\
\hline$v_{\infty}^{u n c}$ & Reversed Gaussian Tail & $3.34[\mathrm{~km} / \mathrm{s}]$ & $25.0[\mathrm{~m} / \mathrm{s}]$ & {$[3.24,3.34][\mathrm{km} / \mathrm{s}]$} \\
$\alpha_{\infty}^{u n c}$ & Gaussian & $38.39[\mathrm{deg}]$ & $0.250[\mathrm{deg}]$ & {$[37.39,39.39][\mathrm{deg}]$} \\
$\beta_{\infty}^{u n c}$ & Gaussian & $-28.68[\mathrm{deg}]$ & $0.125[\mathrm{deg}]$ & {$[-29.18,-28.18][\mathrm{deg}]$} \\
\hline
\end{tabular}

corresponding to the covariance matrix reconstructed from the samples. It is possible to see that, by construction, the distribution is asymmetric, and the mean significantly deviates from the mode, located in the dark red coloured area. Hence, the first two moments are not fully representative of the real distribution. In such a case, the method implemented in IPANeMA is further justified as it does not require any hypothesis on the shape of the distribution or the linearity of the dynamics.

This optimal control problem under uncertainty is formulated by substituting the final boundary condition with a probability constraint. Specifically, the probability that the final position and velocity have a difference, $\Delta r$ and $\Delta v$, with respect to the target position and velocity, smaller than or equal to the two thresholds $\Delta r_{\text {thres }}$ and $\Delta v_{\text {thres. }}$. The values of the two thresholds are defined in Table 3 .

Table 3: Position and velocity threshold for probability constraint.

\begin{tabular}{c|l}
\hline Deviation & Value \\
\hline$\Delta r_{\text {thres }}$ & $3.0 e 5[\mathrm{~km}]$ \\
$\Delta v_{\text {thres }}$ & $100.0[\mathrm{~m} / \mathrm{s}]$ \\
\hline
\end{tabular}

For a given state realisation $\mathbf{x}$, the probability constraint is then computed via 

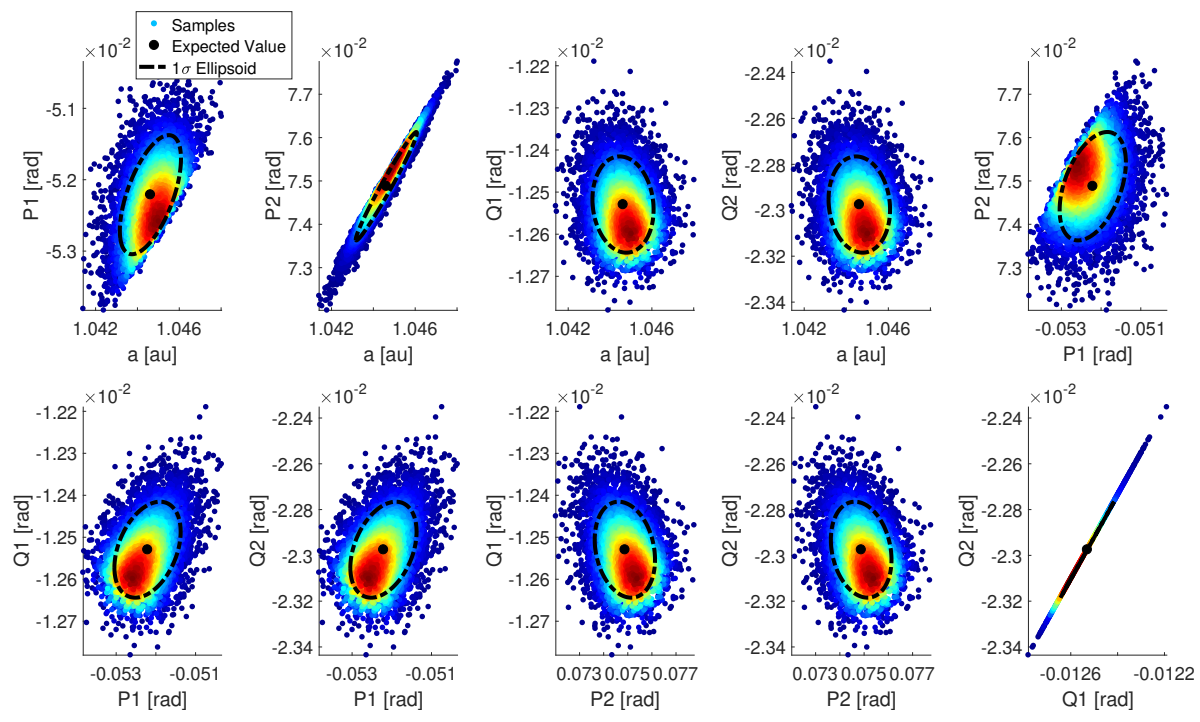

Figure 3: Initial projections of the distribution of equinoctial elements induced by the uncertainty in the hyperbolic excess velocity at departure. The black dot is the expected value and the dashed line is the boundary of the $1 \sigma$ ellipsoid associated to the sample distribution. The color code indicates the probability density associated to each sample.

the auxiliary positive continuous function:

$$
\eta_{T}(\mathbf{X}=\mathbf{x})= \begin{cases}\leq 1 & \text { if } \Delta r \leq \Delta r_{\text {thres }} \wedge \Delta v \leq \Delta v_{\text {thres }} \\ >1 & \text { if } \Delta r>\Delta r_{\text {thres }} \vee \Delta v>\Delta v_{\text {thres }},\end{cases}
$$

and by setting the probability threshold $1-\rho$ to $95 \%$. A quadratic smoothing function is used for the convolution operator [13]:

$$
h\left(\eta_{T}(\mathbf{x})\right)=3\left(1-\eta_{T}(\mathbf{x})^{2}\right) \mathbb{I}_{[-1,+1]} / 4 .
$$

Uncertainty is propagated with 4-degree Chebyshev polynomials, given their proven superior global convergence properties [11, 12]. Degree 4 has been selected as suitable trade-off between representation accuracy (see Section 4.4) and numerical complexity for the intrusive propagation. The fourth order RungeKutta numerical scheme is employed to integrate the dynamical equations of motion. For the transcription with multiple shooting 6 equally sized time intervals, constant controls on each interval and 1000 samples for the calculation of 


\subsection{Results}

The optimised robust control profile components are shown in Figure 4 and compared to the control profile of the deterministic solution. While the thrust
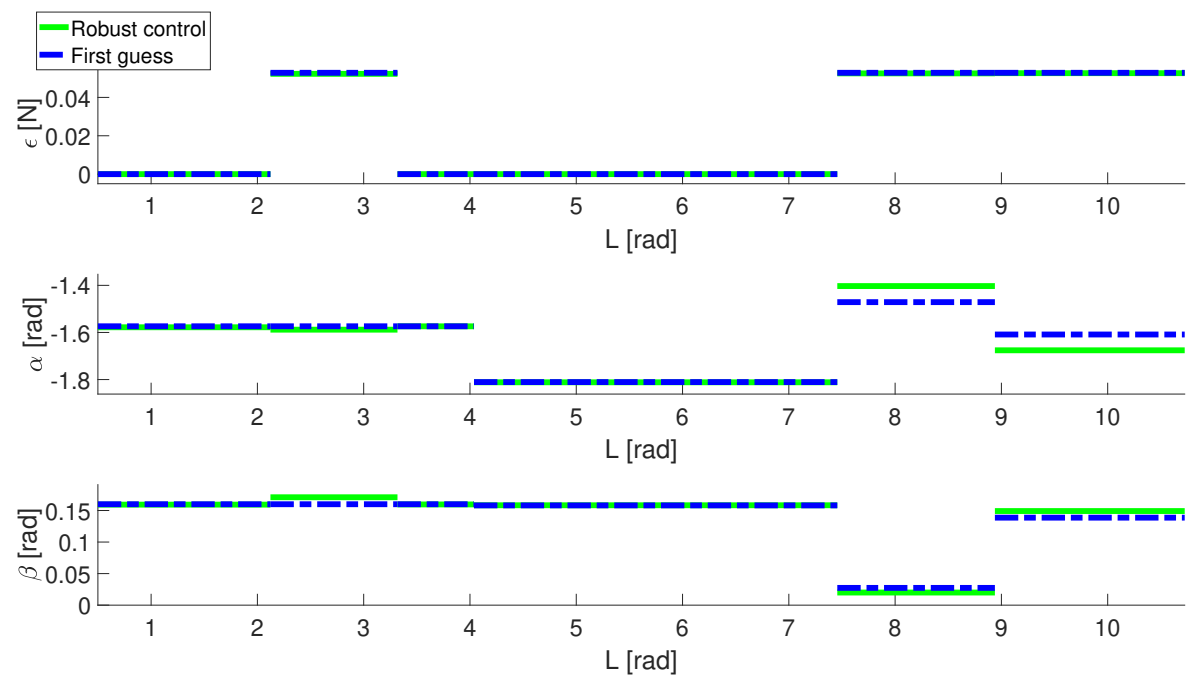

Figure 4: Optimised robust control profile and deterministic control components.

magnitude is essentially unaltered, the thrust angles changed significantly to steer the set of final states within the required target region. In fact the robust solution achieves a probability of $95.60 \%$ that the final state is within the target region. On the contrary when the robust control profile is replaced with the control profile of the deterministic reference trajectory, the probability of meeting the final target region drops to $69.80 \%$.

Figure 5 shows the probability to have a deviation in the final state that is lower than a given threshold (in position and velocity). The blue dash-dot line is the cumulative probability associated to the control profile of the deterministic reference solution, while the green solid line represents the cumulative probability of the optimal robust solution. The 0.95-quantile is highlighted for both the distributions to show how the robust solution satisfies the probabilistic 

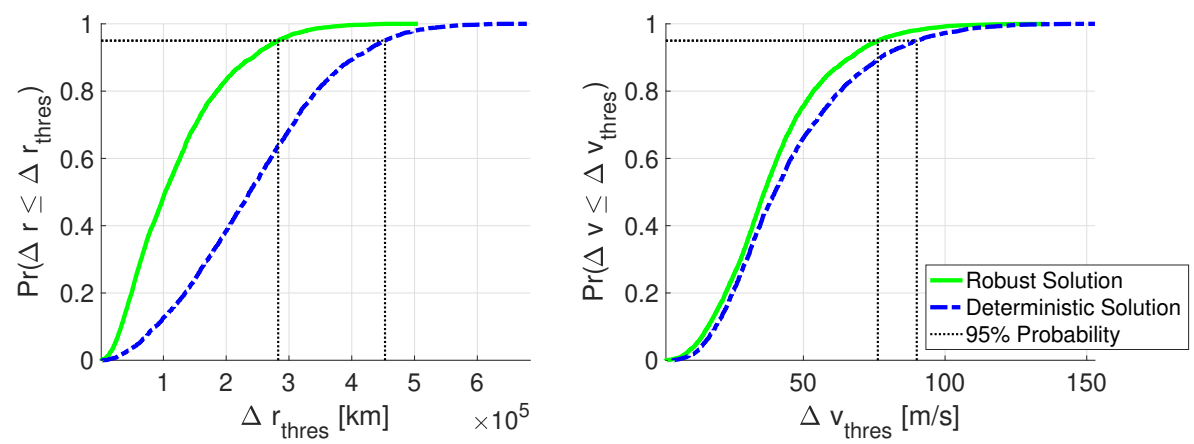

Figure 5: Cumulative distribution functions of final position and velocity deviations for robust and deterministic reference solution.

threshold with a significantly lower constraint deviation. Furthermore, the figure shows that robust distribution always has a higher cumulative probability value for any threshold. This demonstrates that the robust solution probabilistically outperforms the deterministic control for every possible value of the deviation thresholds.

The three-dimensional interplanetary trajectory, corresponding to the peak values of the injection uncertain conditions in Table 2 is shown in Figure 6 . decomposed in its ecliptic in-plane and out-of-plane projections.

\subsection{Validation and Verification}

In this section we verify the accuracy of the two key approximations employed in the optimisation routine: the propagation with GPA, and the probability approximation by convolution on a limited number of samples.

To this end, $10^{5}$ samples drawn from the initial distribution are propagated forward in time with the robust control profile, either using the polynomial approximation coming from GPA or by direct numerical integration of the dynamics with a fourth order Runge-Kutta integrator. The resulting root-mean-square (RMS) of the difference between the GPA approximation and the numerical integration of the equinoctial elements, at the final time, is reported in Table 4 These RMS deviations result in an error of $10^{-4}$ relative to the threshold values as set in Table 3. Therefore, these results confirm that GPA produces a fully 


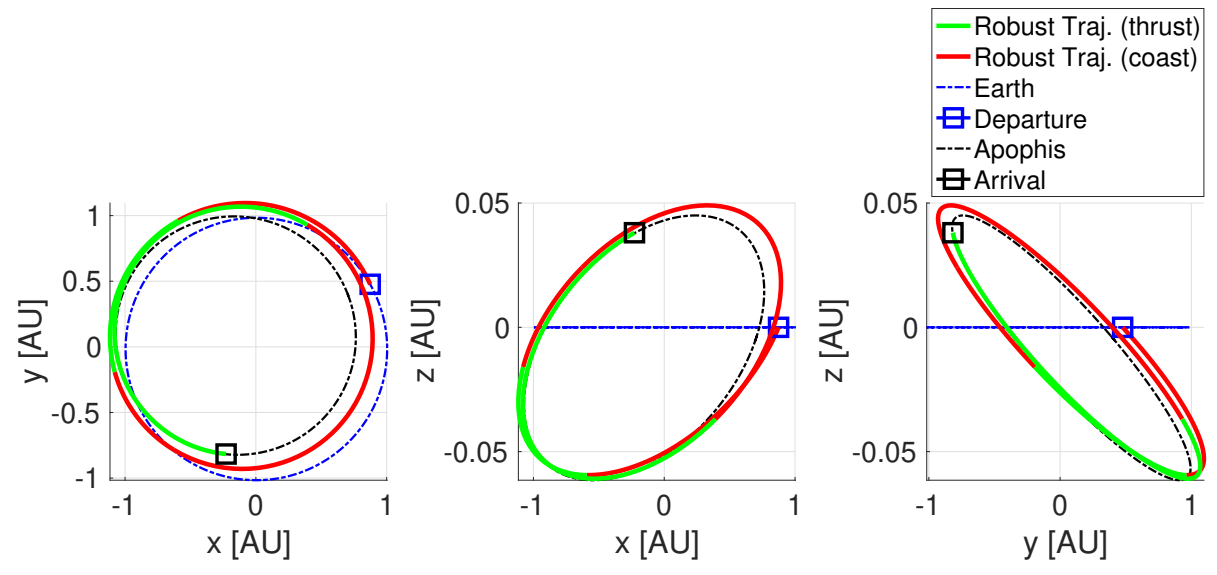

Figure 6: Three-dimensional robust interplanetary trajectory of spacecraft departing from Earth and arriving at Apophis with initial conditions resulting from the peak values of the uncertainties in Table 2 The green and red lines represent the spacecraft trajectory during thrust and coast arcs respectively.

satisfactory propagation approximation as required for computing accurately the probability constraint.

Table 4: Root-mean-square error per state component between $10^{5}$ samples propagated by numerical integration and by intrusive polynomial algebra.

\begin{tabular}{c|c|c|c|c}
\hline$a[\mathrm{AU}]$ & $P_{1}[\mathrm{deg}]$ & $P_{2}[\mathrm{deg}]$ & $Q_{1}[\mathrm{deg}]$ & $Q_{2}[\mathrm{deg}]$ \\
\hline $1.17 \cdot 10^{-6}$ & $3.80 \cdot 10^{-6}$ & $1.88 \cdot 10^{-5}$ & $4.37 \cdot 10^{-6}$ & $8.88 \cdot 10^{-6}$ \\
\hline
\end{tabular}

Then, the numerically propagated samples are used to compute the probability of hitting the final target region, without the convolution approximation. The result is $95.97 \%$, slightly higher than the value $95.60 \%$ that was calculated in the optimisation loop. Table 5 reports the probability resulting from different combinations of control profiles, propagation schemes, number of samples, and with or without the convolution operator.

The results on lines two and four of Table 5 confirm that the use of GPA and the convolution function provide a very good approximation of the probability 
Table 5: Probability of matching the final target region for different control laws and approximation schemes.

\begin{tabular}{c|c|c|c|c}
\hline Solution & Propagation & Samples & Convolution & Probability \\
\hline Deterministic & Polynomial & $10^{3}$ & Yes & $69.80 \%$ \\
Robust & Polynomial & $10^{3}$ & Yes & $95.60 \%$ \\
Robust & Polynomial & $10^{5}$ & No & $96.00 \%$ \\
Robust & Numerical & $10^{5}$ & No & $95.97 \%$ \\
\hline
\end{tabular}

of meeting the final target region.

A time comparison is then performed between the average time employed to propagate a sample (the average over the $10^{5}$ samples) with a fourth order Runge-Kutta numerical integrator and the recursive polynomial mapping with GPA. A time step of 2 hours was used in both propagations. The results, averaged out of ten tests on an OptiPlex7050 desktop with Intel(R) Core i7$7700 @ 3.60 \mathrm{GHz}$ with 8GB of RAM running Ubuntu 18.04.1 LTS, are reported in Table 6. The table shows that the polynomial mapping is almost one order of

Table 6: Average time comparison for propagation of a sample with fourth order Runge-Kutta numerical integrator and with polynomial surrogate mapping.

\begin{tabular}{c|c}
\hline Propagation & Average Time $[\mathrm{ms}]$ \\
\hline Polynomial & 1.5 \\
Numerical & 13.4 \\
\hline
\end{tabular}

magnitude less computationally expensive, per propagated sample, than a full numerical integration. Since this time has to be multiplied by the number of samples, and the number of times sampling is required in the optimisation loop, the employment of intrusive polynomial algebra results crucial to the practical solution of realistic scenarios. 


\subsection{Imprecise and Epistemic Uncertainty}

475 uncertainty is epistemic or probabilities are imprecise. When uncertainty is epistemic there is a lack of knowledge on which distribution $p$ has to be used and we can only know that $p$ belongs to a set of possible probability density functions: $p \in \mathcal{P}$. On the other hand when imprecision is affecting probabilities one can argue that the probability of a realisation depends on a family of distributions rather than on a single one. In both cases, in general, the probability distribution $p$ is not exactly known, and one can only know the boundaries of the set $\mathcal{P}$ of possible density functions. In this section we will consider the less general case in which $p$ belongs to a known family of probability distribution with parameters direct Monte Carlo sampling as used in Section 4.2 .

Since the key aspect of handling imprecision and epistemic uncertainty is the computation of the expectation of quantities of interest, in the following we use the robust control optimised previously computed in Section 4.3 and we simply calculate the different cumulative density functions which result from the set $\mathcal{P}$.

For each uncertain parameter, we consider a different family of density functions: a family of reversed Gaussian tail for the norm of the escape velocity and two different families of Gaussian for azimuth and elevation angles (see Table 7). Epistemic uncertainty affects the knowledge of the standard deviation of these distributions, which is now defined as an interval. By construction, the precise uncertainty in Table 2 falls within the imprecise set.

A three dimensional uniform density function over the defined support is used as proposal distribution

$$
\pi(\boldsymbol{\xi})=\mathcal{U}_{[3.24,3.34]}\left(v_{\infty}^{\text {unc }}\right) \cdot \mathcal{U}_{[37.39,39.39]}\left(\alpha_{\infty}^{\text {unc }}\right) \cdot \mathcal{U}_{[-29.18,-28.18]}\left(\beta_{\infty}^{\text {unc }}\right)
$$


Table 7: Parameterisation of imprecise set of density functions $\mathcal{P}$ modelling uncertainties on the hyperbolic excess velocity at departure from Earth for the same supports defined in Table 2

\begin{tabular}{c|l|c|c}
\hline Component & Density Function & Param. & Std. deviation \\
\hline$v_{\infty}^{\text {unc }}$ & Reversed Gaussian Tail & $3.34[\mathrm{~km} / \mathrm{s}]$ & {$[20.0,33.3][\mathrm{m} / \mathrm{s}]$} \\
$\alpha_{\infty}^{u n c}$ & Gaussian & $38.39[\mathrm{deg}]$ & {$[0.2,0.33][\mathrm{deg}]$} \\
$\beta_{\infty}^{u n c}$ & Gaussian & $-28.68[\mathrm{deg}]$ & {$[0.1,0.166][\mathrm{deg}]$} \\
\hline
\end{tabular}

to draw samples. The samples are propagated only once, and then the expectations corresponding to different admissible densities $p \in \mathcal{P}$ are computed with different weights as in Equation (19). The empirical cumulative distribution functions for a number of distributions $p$ are shown in Figure 7 (dash-dot line with different markers). For comparison, also the CDF corresponding to the robust solution in Figure 5 is added to the figure (green solid line). Given the construction of $\mathcal{P}$, the robust solution falls within the set of empirical CDFs.
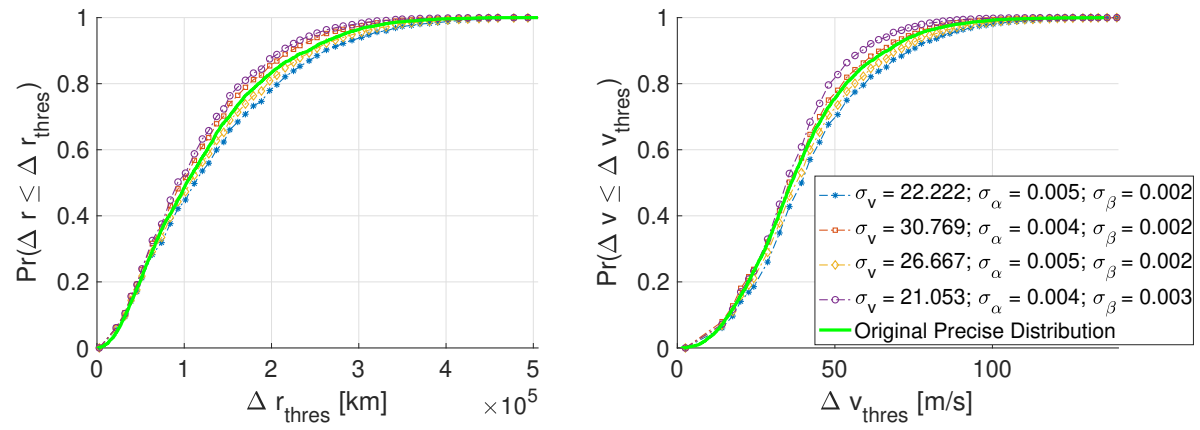

Figure 7: Empirical cumulative distribution functions of final position and velocity deviations for different uncertainty density functions out of the imprecise set $\mathcal{P}$ and for the original precise distribution defined in Table 2

Note that the use of a set of distributions rather than a single one does not affect the transcription and propagation schemes. However, the NLP solver needs to evaluate only one expectation function. Hence, one should replace the expectation functions in problem 10 with either the lower or upper expectation 
functions defined as follows

$$
\begin{aligned}
& \underline{\mathbb{E}}[\phi]:=\inf _{p \in \mathcal{P}} \mathbb{E}_{p}[\phi] \\
& \overline{\mathbb{E}}[\phi]:=\sup _{p \in \mathcal{P}} \mathbb{E}_{p}[\phi] .
\end{aligned}
$$

For the constraint in probability defined in Table 3 , the idea is to evaluate the lowest probability of satisfying the constraint, given the set $\mathcal{P}$, which corresponds to the worst case constraint violation. Therefore, the constraint would be imposed on the lower expectation:

$$
\underline{\mathbb{E}}\left[\mathbb{I}_{T}\left(\mathbf{X}_{f}\right)\right]=\inf _{p \in \mathcal{P}} \operatorname{Pr}\left(\mathbf{X}_{f} \in T\right) \in[1-\rho, 1]
$$

\section{Conclusions}

The paper introduced a novel direct transcription method for the solution of optimal control problems under uncertainties of different nature.

The formulation proposed in this paper can accommodate different forms of the quantities of interest and does not require any underlying assumption on the model used to quantify uncertainty. In fact, it was shown that it can incorporate also epistemic uncertainties and imprecision without any modification of the transcription scheme.

The novel transcription method, implemented in the tool IPANeMA, integrates a multiple shooting numerical scheme with a generalised polynomial expansion to represent and propagate uncertainty regions. A new reinitialisation and recovery approach was introduced to limit the dimensionality of the uncertain space, across multiple time segments, which intrinsically satisfies the continuity conditions between two adjacent, and removes the need of additional constraints. A specific convolution approach was implemented to avoid discontinuities in the evaluation of objective and constraints and allow the use of gradient-based methods.

IPANeMA is capable of handling both uncertainty in the initial state and in the model parameters. Furthermore, IPANeMA is suitable to work with a 
large variety of uncertainty models, hence it is not restricted to purely Gaussian, uniform or other basic probability distribution families.

In this paper, IPANeMA was successfully applied to the robust optimisation subject to uncertain hyperbolic excess velocity at departure. The resulting robust control law achieved over $95 \%$ of probability to reach the final target set. Furthermore, it was shown that this robust solution significantly outperforms the reference, deterministic, one.

It was also shown that the method for the propagation of uncertainty and the calculation of the expectation is fast and accurate, and has a low computational complexity. In particular, it was shown that the polynomial mapping is very accurate when compared to a direct numerically integration of the dynamics, and the convolution operator produces an accurate estimation of the correct 540 probability.

Current developments of IPANeMA aim at incorporating epistemic uncertainty and imprecision in the optimisation and include observations and state estimations for the computation of an updated control. The developed framework seems to suit naturally an interface with a particle filter.

${ }_{545}$ Acknowledgements

This work was partially funded by the European Commission's H2020 programme, through the H2020-MSCA-ITN-2016 UTOPIAE Marie Curie Innovative Training Network, grant agreement 722734 .

\section{References}

\section{References}

[1] A. E. Bryson, Y. C. Ho, Applied optimal control: optimization, estimation, and control, Routledge, New York, 1975. doi:10.1201/9781315137667. 
[2] D. Grebow, G. J. Whiffen, D. Han, B. Kennedy, Dawn safing approach to Ceres re-design, in: AIAA/AAS Astrodynamics Specialist Conference, 2016. doi:10.2514/6.2016-5426.

[3] H. Kuninaka, K. Nishiyama, Y. Shimizu, I. Funaki, H. Koizumi, S. Hosoda, D. Nakata, Hayabusa asteroid explorer powered by ion engines on the way to earth, in: Proceedings of the 31st International Electric Propulsion Conference, 2009.

[4] H. Kuninaka, J. I. Kawaguchi, Lessons learned from round trip of Hayabusa asteroid explorer in deep space, in: 2011 Aerospace Conference, IEEE, 2011, pp. 1-8. doi:10.1109/AERO.2011.5747599.

[5] M. D. Rayman, S. Williams, Design of the first interplanetary solar electric propulsion mission, Journal of Spacecraft and Rockets 3 (2002) 589-595. doi: $10.2514 / 2.3848$

[6] A. Richards, J. How, Robust stable model predictive control with constraint tightening, in: American Control Conference 2006 IEEE, 2006. doi:10. 1109/ACC.2006.1656440.

[7] E. D. Gustafson, Stochastic optimal control of spacecraft, Ph.D. thesis, The University of Michigan (2010). doi:2027.42/77707.

[8] P. Di Lizia, R. Armellin, F. Bernelli-Zazzera, M. Berz, High order optimal control of space trajectories with uncertain boundary conditions, Acta Astronautica 93 (2014) 217-229. doi:10.1016/j.actaastro.2013.07.007.

[9] N. Ozaki, S. Campagnola, R. Funase, C. H. Yam, Stochastic differential dynamic programming with unscented transform for low-thrust trajectory design, Journal of Guidance, Control, and Dynamics 41 (2018) 377-387. doi:10.2514/1.G002367,

[10] M. Vasile, C. Ortega Absil, A. Riccardi, Set propagation in dynamical systems with generalised polynomial algebra and its computational com- 
plexity, Communications in Nonlinear Science and Numerical Simulation 75 (2019) 22-49. doi:10.1016/j.cnsns.2019.03.019

[11] A. Riccardi, C. Tardioli, M. Vasile, An intrusive approach to uncertainty propagation in orbital mechanics based on Tchebycheff polynomial algebra, Advances in Astronautical Sciences (2015) 707-722. URL https://strathprints.strath.ac.uk/60560/

[12] C. A. Ortega, R. Serra, A. Riccardi, M. Vasile, De-orbiting and re-entry analysis with generalised intrusive polynomial expansions, in: 67th International Astronautical Congress, 2016.

URL https://strathprints.strath.ac.uk/60596/

[13] L. Andrieu, G. Cohen, F. Vázquez-Abad, Stochastic programming with probability constraints, arXiv preprint arXiv:0708.0281.

[14] J. T. Betts, Practical methods for optimal control and estimation using nonlinear programming, Vol. 19, Siam, 2010. doi:10.1137/1.9780898718577.

[15] C. A. Ortega, A. Riccardi, M. Vasile, C. Tardioli, SMART-UQ: Uncertainty

595 Q Quantification Toolbox for Generalised Intrusive and non Intrusive Polynomial Algebra, in: 6th International Conference on Astrodynamics Tools and Techniques, 2016.

URL https://strathprints.strath.ac.uk/58920/

[16] T. Augustin, F. P. A. Coolen, G. De Cooman, M. Troffaes, Introduc600 tion to Imprecise Probabilities, John Wiley \& Sons, 2014. doi:10.1002/ 9781118763117

[17] A. Doucet, N. De Freitas, N. Gordon, Sequential Monte Carlo Metha ods in Practice, 1st Edition, Springer, New York, 2001. doi:10.1007/ 978-1-4757-3437-9.

[18] C. Büskens, D. Wassel, The ESA NLP-Solver WORHP, in: G. Fasano, J. Pintér (Eds.), Modeling and Optimization in Space Engineering, 
Vol. 73, Springer, New York, NY, 2012, pp. 85-110. doi:10.1007/ 978-1-4614-4469-5_4.

[19] C. Acton, N. Bachman, B. Semenov, E. Wright, A look toward the future in the handling of space science mission geometry, Planetary and Space Science 150 (2018) 9-12. doi:10.1016/j.pss.2017.02.013

[20] R. Broucke, P. J. Cefola, On the equinoctial orbit elements, Celestial Mechanics 5 (3) (1972) 303-310. doi:10.1007/BF01228432.

[21] F. Zuiani, M. Vasile, Extended analytical formulas for the perturbed Keplerian motion under a constant control acceleration, Celestial Mechanics and Dynamical Astronomy 121 (2015) 275-300. doi:10.1007/ s10569-014-9600-5.

[22] M. Di Carlo, J. M. Romero Martin, M. Vasile, CAMELOT: ComputationalAnalytical Multi-fidElity Low-thrust Optimisation Toolbox, CEAS Space Journal 10 (2018) 25-36. doi:10.1007/s12567-017-0172-6. 